\title{
Psychiatric and sleep abnormalities in school-age children with migraine
}

\author{
Yasser Abo Elfotoh El-Heneedy ${ }^{1}$, Wafik Said Bahnasy ${ }^{1 *}$ (1), Shereen Ahmed ELAhwal', Reham Abdel Rahman Amer², \\ Shereen Desoky Ahmed Abohammar ${ }^{2}$ and Heba Ahmed Mohamed Salem ${ }^{3}$
}

\begin{abstract}
Background: Migraine is a primary headache that commonly starts in childhood and adolescent's periods with great negative impacts on the educational and psychosocial performances of its sufferers. The objectives of this work were to study the existence and types of sleep and psychiatric abnormalities in school-age children with migraine (SCM).
\end{abstract}

Methods: The study was conducted on 40 SCM and 20 age- and sex-matched healthy control subjects (HCS) submitted to history taking, neurological examination, Pediatric Migraine Disability Assessment (PedMIDAS) questionnaire, Child Behavior Checklist (CBCL), Epworth Sleepiness Scale for Children and Adolescents (ESS-CHAD), Pittsburgh Sleep Quality Index (PSQI) and one-night polysomnography (PSG).

Results: The study showed a high incidence of psychiatric and sleep abnormalities in SCM. The most common psychiatric disorders were anxious depressed symptoms, withdrawal depressed symptoms, social problem, somatic complaints, and attention problems. At the same time, SCM experienced decreased sleep quality, excessive daytime sleepiness (EDS), and PSG abnormalities in the form of decreased total sleep time (TST) and sleep efficiency (SE) in addition to increased sleep latency, wake after sleep onset (WASO), arousal index (Al), and REM sleep without atonia index.

Conclusion: Sleep and psychiatric abnormalities are common underdiagnosed pediatric migraine comorbidities greatly reducing headache control and school performance in this very important period of psychosocial development.

Keywords: Pediatric migraine, School-age children, Child Behavior Checklist, PedMIDAS, Polysomnogram

\section{Introduction}

Pediatric migraine is one of the primary headache disorders that commonly met in the pediatric headache clinic and emergency units that may occur at any age even infancy. Migraine is characterized by complex neurovascular disturbances and brain sensory hyperexcitability leading to recurrent attacks of severe, throbbing headache (often unilateral), lasting 4-72 h, accompanied by nausea, vomiting, and extreme sensitivity to light and sound [1]. Childhood migraine is usually genetically determined affecting 1-3\% of children below the age of 7

\footnotetext{
* Correspondence: wafiq.elbahnasi@med.tanta.edu.eg

The work was carried out in The Neuropsychiatry and Chest Departments,

Tanta University Hospitals, Faculty of Medicine, Tanta University, zip code

31527, Tanta, Egypt.

${ }^{1}$ Neurology Unit, Neuropsychiatry Department, Tanta University, Tanta 31527,

Egypt

Full list of author information is available at the end of the article
}

years and $8-23 \%$ of adolescents. Boys are slightly more affected than girls before puberty, but around the age of puberty, the ratio changes to be more common in girls [2]. Types of pediatric migraine include migraine with aura, migraine without aura and basilar, confusional as well as hemiplegic migraine [3].

Pediatric migraine is commonly associated with many psychiatric disorders including separation anxiety disorders, school phobia, enuresis, and encopresis as well as adjustment, conduct, and learning disorders [4]. These psychiatric disturbances have marked negative impacts on headache control, psycho-social competence, and quality of life [5]. There is a bidirectional relationship between childhood migraine and sleep disturbances possibly due to sharing the same neuropeptides abnormalities including serotonin, dopamine, and/or melatonin [6]. Migraine sufferers often have trouble falling asleep, 
insufficient sleep, bedwetting, somnophobia, parasomnias, and excessive daytime sleepiness (EDS) which negatively influence the child's health and intellectual performance $[7,8]$.

\section{Aim of the work}

The aim of this work was to study the existence and types of sleep and psychiatric abnormalities in school-age children with migraine.

\section{Methods}

The present study was a case control prospective one conducted on $40 \mathrm{SCM}$ (group I) and 20 age- and sex-matched healthy control subjects (HCS) (group II) attending the outpatient headache clinic of the Neurology Department, Tanta University Hospitals, in the period from 1st of March 2017 to the end of May 2018. Migraine was diagnosed according to The International Classification of Headache Disorders, 3rd edition (ICHD-3) [9].

Exclusion criteria included children with premorbid psychiatric problems, mental disorders, and nocturnal enuresis and those who recently received drugs (e.g., antihistaminic drugs, systemic bronchodilators, benzodiazepines sedative drugs, or tricyclic antidepressants) or suffered from any medical disorders affecting sleep. Children with brain MRI abnormalities were also excluded which was performed to exclude secondary headache causes using 1.5-Tesla, General Electric Scanner, GE Medical System, Milwaukee, WI, USA.

The study's protocol was approved by The Research Ethics Committee and Quality Assurance Unit, Faculty of Medicine, Tanta University. Participations were voluntary, informed consents were approved by all participants' guardians, and any possible risks were clarified.

Included subjects were submitted to history taking (stressing on the family history of migraine), neurological examination, and the Pediatric Migraine Disability Assessment questionnaire (PedMIDAS) [10] to evaluate the headache-induced disabilities.

Psychiatric assessments were done using the Arabic-translated and validated versions of the Child Behavior Checklist (CBCL) which were also completed by a parent and scored using a computerized scoring software system (Assessment Data Manager-version 9.1) $[11,12]$ (Additional file 1).

Included subjects were submitted to sleep evaluation using the Epworth Sleepiness Scale for Children and Adolescents (ESS-CHAD) [13] and Pittsburgh Sleep Quality Index (PSQI) (https://www.opapc.com/uploads/documents/PSQI.pdf). One-night polysomnography (PSG) was done for all included subjects using Somon Medics $\mathrm{GmbH}$ (Am SonnenstuhL63, D-97236 Rander Sacker, Type: SOMNO screenTMplus, SN: 4259, kw45: 2014,
Germany). The PSG parameters were scored according to The American Academy of Sleep Medicine Manual for the Scoring of Sleep and Associated Events: Rules, Terminology and Technical Specifications, version 2.4 [14] (Additional file 2).

Statistical analysis of the collected data was done using SPSS Prism, version 20, 2013, created by IBM, Chicago, IL, USA. Differences between studied groups were done by the Student $t$ test and chi-square. Correlation analysis was performed using Pearson's correlation coefficient. $P$ value $<0.05$ was considered statistically significant.

\section{Results}

Demographic data of the included patients showed that their age was $11.13 \pm 2.85$ years, approximate age of migraine onset $9.23 \pm 1.03,23 / 40(57.5 \%)$ were females, 17 / 40 (42.5\%) were males, and 12/40 (30\%) had positive family history of migraine (either affected parent or sibling). The PedMIDAS of the included SCM was $51.95 \pm$ 7.81 points which means that the headache caused moderate to severe disabilities to patients in exerting their daily activities. Regarding migraine subtypes, 26/40 (65\%) had migraine without aura, 11/40 (27.5\%) had migraine with aura $(5 / 11$ had visual and $6 / 11$ had abdominal auras) and 3/40 (7.5\%) had chronic migraine.

Psychiatric assessment using the CBCL for the included subjects revealed that there were statistically significant increases in each of anxious depressed symptoms, withdrawal depressed symptoms, social problem, somatic complaints, and attention problems in SCM compared to HCS with $p$ values $<0.05$. On the other hand, thought problems and delinquent and aggressive behaviors showed statistically non-significant differences with $p$ values $>0.05$ (Table 1 ). The PedMIDAS showed strong positive correlation with the CBCL anxious depressed symptoms (Fig. 1) but showed no correlations with each of withdrawal depressed symptoms,

Table 1 Statistical analysis of the Child Behavior Checklist among school children with migraine (group I) and healthy control subjects (group II)

\begin{tabular}{|c|c|c|c|c|}
\hline & \multirow{2}{*}{$\begin{array}{l}\text { Group I } \\
\text { Mean } \pm \text { SD }\end{array}$} & \multirow{2}{*}{$\begin{array}{l}\text { Group II } \\
\text { Mean } \pm \text { SD }\end{array}$} & \multicolumn{2}{|l|}{$t$ test } \\
\hline & & & $t$ value & $p$ value \\
\hline Anxious/depressed & $67.18 \pm 4.67$ & $58.70 \pm 4.38$ & 16.985 & $0.001^{*}$ \\
\hline Withdrawn/depressed & $65.70 \pm 9.73$ & $58.60 \pm 5.63$ & 9.072 & $0.004^{*}$ \\
\hline Social problems & $64.13 \pm 8.44$ & $58.00 \pm 5.07$ & 8.877 & $0.004^{*}$ \\
\hline Somatic complaint & $64.38 \pm 9.49$ & $58.15 \pm 5.10$ & 7.481 & $0.008^{*}$ \\
\hline Attention problems & $62.78 \pm 9.27$ & $53.80 \pm 4.31$ & 16.808 & $0.001^{*}$ \\
\hline Thought problems & $55.00 \pm 3.49$ & $55.45 \pm 3.05$ & 0.241 & 0.626 \\
\hline Delinquent behavior & $55.28 \pm 3.63$ & $54.85 \pm 3.63$ & 0.183 & 0.671 \\
\hline Aggressive behaviors & $56.45 \pm 3.46$ & $54.80 \pm 4.14$ & 2.655 & 0.109 \\
\hline
\end{tabular}

*Significant 

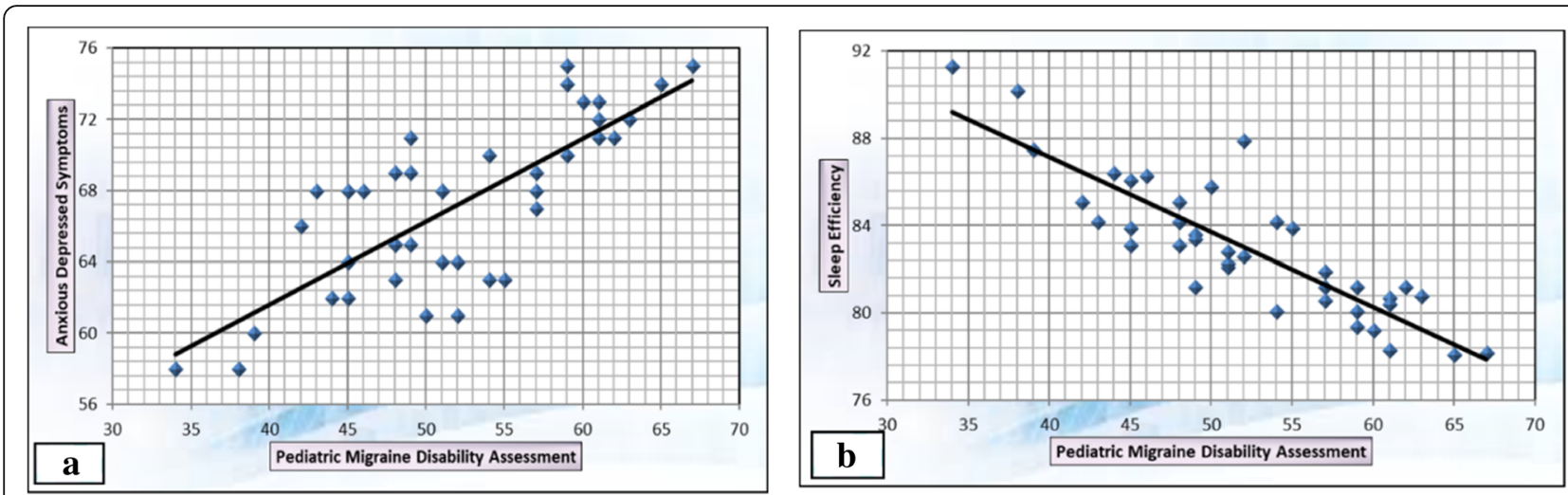

Fig. 1 a Positive correlation between PedMIDAS and anxious/depressed symptoms. b Negative correlation between PedMEDAS and sleep efficiency. PedMIDAS pediatric migraine disability assessment scale

social problem, somatic complaints, and attention problems.

Sleep assessment results revealed that SCM had significant increase in each of ESS-CHAD and PSQI compared to HCS with $p$ value $<0.05$. Regarding PSG parameters, there were statistically significant decrease in each of total sleep time (TST), sleep efficiency (SE), and REM percent of TST in SCM compared to HCS with $p$ values $<0.05$ (Table 2). At the same time, there were statistically significant increase in each of sleep latency (SL), wake after sleep onset (WASO), sleep stage transition index (SSTI), REM sleep without atonia (RSWA) index, N3 percent of TST, arousal index (AI), snore index (SI), and periodic limb movement (PLMs) index in SCM than HCS with $p$ values $<0.05$. Each of REM latency, $\mathrm{N} 1$ and N2 percent of TST, and apnea hypopnea index (AHI) showed statistically non-significant differences between the two studied groups with $p$ values $>0.05$ (Table 2 and Fig. 2). The PedMIDAS was positively correlated with each of WASO, SSTI, and AI as well as PLMs index and negatively correlated with TST and SE (Fig. 1).

Table 2 Statistical analysis of the studied sleep scales and polysomnographic results among school children with migraine (group I) and healthy control subjects (group II)

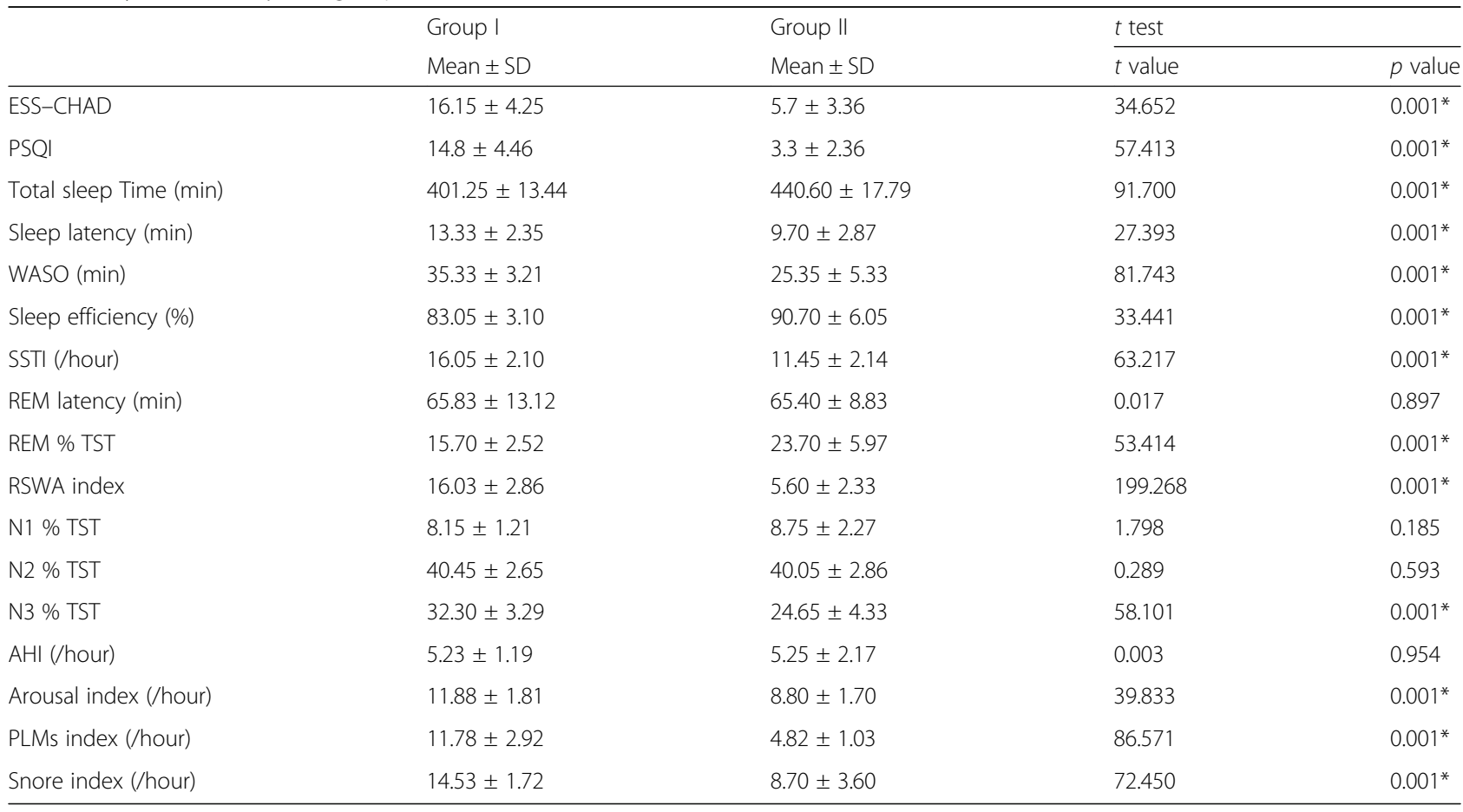

AHI apnea hypopnea index; ESS-CHAD Epworth Sleepiness Scale for Children and Adolescents; N1, N2, N3 non-rapid eye movement stages 1, 2, 3; PLMs periodic limb movements; PSQI Pittsburgh Sleep Quality Index; REM rapid eye movement; RSWA REM sleep without atonia; SSTI sleep stage transition index; TST total sleep time; WASO wake after sleep onset

*Significant 

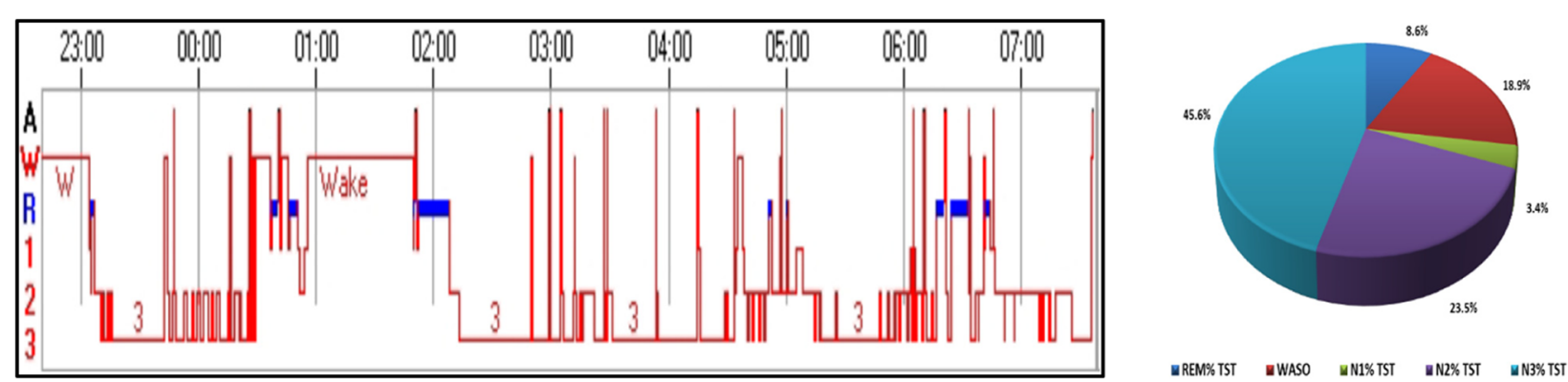

Fig. 2 Shortening of REM \% TST and increased sleep fragmentation and waking after sleep onset

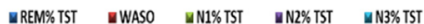

\section{Discussion}

The results of the present study showed that the onset of pediatric migraine is usually around the pubertal age with non-significant sex difference in its incidence during this period despite the well-known higher female prevalence in studies carried out on adult onset migraine pointing to the possible role of female gonadal sex hormones in migraine pathogenesis. At the same time, the study revealed a high incidence of positive family history among migraineurs children pointing to the possible genetic role in its pathogenesis. These results are in harmony with Jin et al. [15] as well as Timothy et al. [16] who found slight non-significant higher incidence of migraine among pre-pubertal boys with strong link between gonadal female sex hormones and migraine development in post-pubertal women. On the other hand, Recober et al. 2018 [17] found higher pediatric migraine female incidence among their studied patients possibly due to higher post-pubertal included patients and exerting their study on patients who required hospital admissions contrasting the outpatient included children of this study. The results of Teleanu et al. [18] documented the high incidence of positive family history migraineurs children, but the incidence in their study is higher than that of the present one ( $60 \%$ vs. $30 \%)$ possibly due to the younger age of their researched children as they worked on the pre-pubertal age.

The study also revealed that pediatric migraine often resulted in moderate to severe disabilities in exerting their usual daily activities with many days of school absenteeism, subnormal performance of household works, and marked disruption of social as well as leisure activities. These results are passing with the work of Akerman et al. [19] who stated that migraine is one of the most common causes of school absenteeism among the pediatric population.

The study also declared that the most common migraine subtype was episodic migraine without aura with a high percent of included patients had positive family history of migraine. These observations are passing with the works of Le et al. [5] as well as Gormley et al. [20] who specified that pediatric migraine tends to run in families with high concordance among monozygotic twins pointing to the inherited nature of the disease.

There is an ultimate bidirectional relation between pediatric migraine and psychological states of the patients. The recurrent migraine attacks negatively affect the child's psycho-social competence while the migraine-associated psychiatric disturbances greatly lessen satisfactory headache control and increase school absenteeism [21]. The study revealed increased anxious-depressed symptoms among SCM which was positively correlated with the disabilities caused by the repeated migraine episodes. At the same time, assessment of other psychiatric parameters revealed competence and behavioral problems among SCM with increased withdrawal depressed symptoms, social problem, somatic complaints, and attention problems with consecutive marked impairment in leisure activities participation and social relations. These results are in harmony with that of Pakalnis et al. [22] as well as Zarea et al. [23] who found increased anxiety and depression scores among migraineurs children compared to HCS which greatly intensify headache episodes and diminish their proper control. They also stated that specified psychiatric therapy is mandatory to SCM for better headache management and educational performance. In a parallel way, Dyb et al. [24] revealed higher incidence of comorbid mood and anxiety disorders (major depressive disorder, panic disorder, generalized anxiety disorder, and specific phobias) in children with migraine. At the same time, Bellini et al. [7] as well as Arruda and Bigal [25] declared significantly higher association of behavioral and internalization symptoms among children and adolescents with migraine especially in those with high headache frequencies.

There is a reciprocal relationship between childhood migraine and sleep disturbances. The nocturnal attacks may disturb sleep while sleep disturbances diminish headache control [26]. The results of the present study revealed a high rate of sleep abnormalities among patients with pediatric migraine which were proportional with the degree of disability caused by the recurrent headache attacks. Children with 
migraine often experience decreased TST and interrupted sleep with difficulty in sleep maintenance (reduced SE and increased WASO as well as SSTI). The PSG also showed deformed sleep architecture (decreased REM percent and increased N3 percent of TST) and distorted REM sleep as well as REM behavior disorders (increased RSWA index). In addition to these disturbances, the increased AI, SI, and PLMs index greatly reduce sleep quality. These results are in accordance with the work of Harnod et al. [6] as well as Tajti et al. [27] who found abnormalities in sleep regulating neuropeptide secretions especially orexin and melatonin in children with migraine. The headache attacks could be triggered by the stimuli that activate the hypothalamic orexin system, such as stress, fatigue, and sleep deprivation. In a parallel way, Karthik et al. 2012 [28] found a high prevalence of sleep disturbances in migraine patients without aura including difficulty in sleep initiation, poor sleep quality, EDS, and early morning awakening. Heyer et al. [29] are also in harmony with the higher incidence of sleep disturbances among children and adolescents with migraine which result in lowered sense of wellbeing, decreased ability to cope with the headache, impaired daytime functioning, and great negative impacts on school performance as well as attendance. Harnod et al. [6] as well as Acar et al. [30] recorded high incidences of malformed sleep microstructure and various types of parasomnias (sleep talking, somnambulism and bruxism) as well as increased PLMs in migraine patients which were associated with higher headache severities scores and increased disabilities caused by migraine. On the other hand, Harnod et al. [6] revealed a higher incidence of subsequent migraine development in children with non-apneic sleep disorders which suggests that sleep abnormalities may prodrome the headache attacks in some patients.

\section{Conclusion}

Pediatric migraine is often associated with sleep and psychiatric abnormalities which have negative impacts on headache control, school attendance, and psychological competence in this important period of personality development.

\section{Recommendations}

Further researches are needed to assess the best ways for psychiatric and sleep abnormalities management in SCM to reduce health care costs and for better school performance, social relations, and emotional wellbeing.

\section{Limitations}

Absence of complicated and episodic syndromes associated with migraine as well as non-evaluation of the effect of migraine treatment on the sleep and psychiatric abnormalities.

\section{Additional files}

Additional file 1: Child Behavior Checklist. (DOCX 73 kb)

Additional file 2: Polysomnography. (DOCX $35 \mathrm{~kb}$ )

\section{Abbreviations}

ADHD: Attention-deficit hyperactivity disorder; AHI: Apnea hypopnea index; Al: Arousal index; CBCL: Child Behavior Checklist; EDS: Excessive daytime sleepiness; ESS-CHAD: Epworth Sleepiness Scale for Children and Adolescents; HCS: Healthy control subjects; ICHD-3: International Classification of Headache Disorders, 3rd edition; PedMIDAS: Pediatric Migraine Disability Assessment; PLMs: Periodic limb movements; PSG: Polysomnography; PSQI: Pittsburgh Sleep Quality Index; REM: Rapid eye movement; RSWA: REM sleep without atonia; SCM: School-age children with migraine; SE: Sleep efficiency; SI: Snore index; SL: Sleep latency; SSTI: Sleep stage transition index; TST: Total sleep time; WASO: Wake after sleep onset sleep

\section{Acknowledgements}

We would like to thank the pediatric neurology and headache clinic teams, Neuropsychiatry Department, Tanta University Hospitals for their great help in patients' selection and evaluation.

Funding

No funding had been received.

\section{Availability of data and materials}

The datasets used and/or analyzed during the current study are available from the corresponding author on reasonable request.

\section{Authors' contributions}

YAH contributed to the study idea and design, patients' assessment and inclusion, imaging interpretation, statistical analysis, references' collection, and manuscript writing and final approval. WSB contributed to the study's idea, design, patients' selection, polysomnogram interpretation, data collection, statistical analysis, references collection, and manuscript writing, revision, and final approval. SAE contributed to the study's design, patients' selection and examination, statistical analysis, data analysis, and manuscript revision and final approval. RAA contributed to the study's idea and design, patients' selection, psychiatric assessment, data analysis, and manuscript revision and final approval. SDAA contributed to the study's idea and design, patients' selection, psychiatric assessment, data analysis, and manuscript revision and final approval. HAMS contributed to the study's patients' selection, polysomnogram interpretation, data analysis, and manuscript revision and final approval.

\section{Ethics approval and consent to participate}

- The manuscript was approved from The Research Ethics Committee and Quality Assurance Unit, Faculty of Medicine, Tanta University.

- The URL: http://tqac.tanta.edu.eg/new-tqac/ QualityAssuranceUnit@hotmail.com

- Approval Code: 31416/03/17

- Name of the PI: Yasser Abo Elfotoh El-Heneedy.

- Name of the department: Neuropsychiatry.

- Type of the research: promotion research.

- Date of approval: March 2017.

- The study's protocol was approved by The Research Ethics Committee and Quality Assurance Unit, Faculty of Medicine, Tanta University. Participations were voluntary, informed consents were approved by all participants' guardians and any possible risks were clarified.

\section{Consent for publication}

Not applicable.

Competing interests

The authors declare that they have no competing interests. 


\section{Publisher's Note}

Springer Nature remains neutral with regard to jurisdictional claims in published maps and institutional affiliations.

\section{Author details}

${ }^{1}$ Neurology Unit, Neuropsychiatry Department, Tanta University, Tanta 31527, Egypt. ${ }^{2}$ Psychiatry Unit, Neuropsychiatry Department, Tanta University, Tanta 31527, Egypt. ${ }^{3}$ Chest Department, Tanta University, Tanta 31527, Egypt.

Received: 20 July 2018 Accepted: 5 February 2019

Published online: 19 February 2019

\section{References}

1. Lagman-Bartolome AM, Lay C. Pediatric migraine variants: a review of epidemiology, diagnosis, treatment, and outcome. Curr Neurol Neurosci Rep. 2015;15(6):34. https://doi.org/10.1007/s11910-015-0551-3.

2. Woldeamanuel YW, Cowan RP. Migraine affects 1 in 10 people worldwide featuring recent rise: a systematic review and meta-analysis of communitybased studies involving 6 million participants. J Neurol Sci. 2017;372:307-15. https://doi.org/10.1016/j.jns.2016.11.071.

3. Charles $\mathrm{A}$. The pathophysiology of migraine: implications for clinical management. Lancet Neurol. 2018;17(2):174-82. https://doi.org/10.1016/ S1474-4422(17)30435-0.

4. Fielding J, Young S, Martin PR, Waters AM. Headache symptoms consistent with migraine and tension-type headaches in children with anxiety disorders. J Anxiety Disord. 2016;40:76-4. https://doi.org/10.1016/j.janxdis. 2016.04.006

5. Le H, Tfelt-Hansen P, Skytthe A, Kyvik KO, Olesen J. Association between migraine, lifestyle and socioeconomic factors: a population-based crosssectional study. J Headache Pain. 2011;12(2):157-72. https://doi.org/10.1007/ s10194-011-0321-9.

6. Harnod T, Wang YC, Kao CH. Higher risk of developing a subsequent migraine in adults with non-apnea sleep disorders: a nationwide population-based cohort study. Eur J Intern Med. 2015;26(4):232-6. https:// doi.org/10.1016/j.ejim.2015.03.002

7. Bellini B, Panunzi S, Bruni O, Guidetti V. Headache and sleep in children. Curr Pain Headache Rep. 2013;17(6):335. https://doi.org/10.1007/s11916-0130335-x.

8. O'Brien HL, Slater SK. Comorbid psychological conditions in pediatric headache. Semin Pediatr Neurol. 2016;23(1):68-70. https://doi.org/10.1016/j. spen.2016.01.002.

9. Olesen J, Bolay H, Charles A, Evers S, First M, Hershey A, et al. The international classification of headache disorders, $3^{\text {rd }}$ edition. Cephalalgia. 2018;38(1):1-211. https://doi.org/10.1177/0333102417738202.

10. Sampaio Rocha-Filho PA, Hershey AD. Pediatric migraine disability assessment (PedMIDAS): translation into Brazilian Portuguese and crosscultural adaptation. Headache. 2017:57(9):1409-15. https://doi.org/10.1111/ head.13159.

11. Achenbach TM, Dumenci L. Advances in empirically based assessment: revised cross-informant syndromes and new DSM-oriented scales for the CBCL, YSR, and TRF: comment on Lengua, Sadowksi, Friedrich, and Fischer (2001). J Consult Clin Psychol. 2001;69(4):699-702.

12. Bahnasy WS, El-Heneedy YAE, El-Seidy EAS, Ibrahim ISE, Seleem MAH. Primary monosymptomatic nocturnal enuresis: an etiological study. Egypt J Neurol Psychiatr Neurosurg. 2018;45:19. https://doi.org/10.1186/s41983-0180020-4.

13. Janssen KC, Phillipson S, O'Connor J, Johns MW. Validation of the Epworth Sleepiness Scale for children and adolescents using Rasch analysis. Sleep Med. 2017;33:30-5. https://doi.org/10.1016/j.sleep.2017.01.014.

14. Berry RB, Brooks R, Gamaldo C, Harding SM, Lloyd RM, Quan SF, et al. AASM scoring manual updates for 2017 (version 2.4). J Clin Sleep Med. 2017;13(5): 665-6. https://doi.org/10.5664/jcsm.6576.

15. Jin Z, Shi L, Wang YJ, Yang LG, Shi YH, Shen LW, et al. Prevalence of headache among children and adolescents in Shanghai, China. J Clin Neurosci. 2013;20(1):117-21. https://doi.org/10.1016/j.jocn.2012.02.020.

16. Timothy J, Steiner TJ, Lars J, Stovner LJ, Theo Vos T. GBD 2015: migraine is the third cause of disability in under 50s. J Headache Pain. 2016;17(1):104. https://doi.org/10.1186/s10194-016-0699-5.

17. Recober A, Patel PB, Thibault DP, Hill AW, Kaiser EA, Willis AW. Sociodemographic factors associated with hospital care for pediatric migraine- a national study using the kids inpatient dataset. Pediatr Neurol 2018. https://doi.org/10.1016/j.pediatrneurol.2017.10.023 In-Press.

18. Teleanu RI, Vladacenco O, Teleanu DM, Epure DA. Treatment of pediatric migraine: a review. Maedica (Buchar). 2016;11(2):136-43.

19. Akerman S, Romero-Reyes M, Holland PR. Current and novel insights into the neurophysiology of migraine and its implications for therapeutics. Pharmacol Ther. 2017;172:151-70. https://doi.org/10.1016/j.pharmthera.2016. 12.005.

20. Gormley P, Kurki MI, Hiekkala ME, Veerapen K, Mitchell AA, Palta DLP, et al. Common variant burden contributes to the familial aggregation of migraine in 1,589 families. Neuron. 2018;98(4):743-53. https://doi.org/10.1016/j. neuron.2018.04.014.

21. Hsu SC, Wang SJ, Liu CY, Juang YY, Yang CH, Hung Cl. The impact of anxiety and migraine on quality of sleep in patients with major depressive disorder. Compr Psychiatry. 2008;50(2):151-7. https://doi.org/10.1016/j. comppsych.2008.07.002.

22. Pakalnis A, Splaingard M, Splaingard D, Kring D, Colvin A. Serotonin effects on sleep and emotional disorders in adolescent migraine. Headache. 2009; 49(10):1486-92. https://doi.org/10.1111/j.1526-4610.2009.01392.x.

23. Zarea K, Rahmani M, Hassani F, Hakim A. Epidemiology and associated factors of migraine headache among iranian medical students: a descriptive-analytical study. Clin Epidemiol Glob Health. 2017;6(3):109-14. https://doi.org/10.1016/j.cegh.2017.10.002

24. Dyb G, Stensland S, Zwart JA. Psychiatric comorbidity in childhood and adolescence headache. Curr Pain Headache Rep. 2015;19(3):5. https://doi. org/10.1007/s11916-015-0479-y.

25. Arruda MA, Bigal ME. Behavioral and emotional symptoms and primary headaches in children: a population-based study. Cephalalgia. 2012;32(15): 1093-100. https://doi.org/10.1177/0333102412454226.

26. Rains JC, Poceta JS. Sleep-related headaches. Neurol Clin. 2012;30(4):128598. https://doi.org/10.1016/j.ncl.2012.08.014.

27. Tajti J, Szok D, Majláth Z, Tuka B, Csáti A, Vécsei L. Migraine and neuropeptides. Neuropeptides. 2015;52:19-30. https://doi.org/10.1016/j. npep.2015.03.006

28. Karthik N, Kulkarni GB, Taly AB, Rao S, Sinha S. Sleep disturbances in migraine without aura: a questionnaire-based study. J Neurol Sci. 2012; 321(1-2):73-6. https://doi.org/10.1016/j.jns.2012.07.057.

29. Heyer GL, Rose SC, Merison K, Perkins SQ, Lee JEM. Specific headache factors predict sleep disturbances among youth with migraine. Pediatr Neurol. 2014; 51(4):489-93. https://doi.org/10.1016/j.pediatrneurol.2014.07.001.

30. Acar BA, Acar T, Alagöz AN, Karacan A, Varım C, Uyanık MS, et al. Relationship between primary restless legs syndrome and migraine with aura. Kaohsiung J Med Sci. 2016;32(8):420-6. https://doi.org/10.1016/j.kjms. 2016.06.003.

\section{Submit your manuscript to a SpringerOpen ${ }^{\circ}$ journal and benefit from:}

- Convenient online submission

- Rigorous peer review

- Open access: articles freely available online

- High visibility within the field

- Retaining the copyright to your article

Submit your next manuscript at $>$ springeropen.com 